\title{
Luteolin, a novel p90 ribosomal S6 kinase inhibitor, suppresses proliferation and migration in leukemia cells
}

\author{
LAN DENG $^{1,2^{*}}$, LING JIANG $^{3 *}$, XIANGHUA LIN ${ }^{1 *}$, KUO-FU TSENG ${ }^{4}$, ZHIGANG LU $^{2}$ and XIUJU WANG ${ }^{1}$ \\ ${ }^{1}$ Department of Hematology, Sun Yat-Sen Memorial Hospital, Sun Yat-Sen University, Guangzhou, Guangdong 510120; \\ ${ }^{2}$ Department of Hematology, Zhujiang Hospital, Southern Medical University, Guangzhou, Guangdong 501282; \\ ${ }^{3}$ Department of Hematology, Nanfang Hospital, Southern Medical University, Guangzhou, Guangdong 510515, P.R. China; \\ ${ }^{4}$ Department of Biophysics, Oregon State University, Corvallis, OR 97330, USA
}

Received August 11, 2015; Accepted November 1, 2016

DOI: $10.3892 / 01.2017 .5597$

\begin{abstract}
Ribosomal S6 kinases (RSKs) are directly regulated by extracellular signal-regulated kinase (ERK) signaling and are implicated in cell growth, survival, motility and senescence. The present study observed that RSK1 was overexpressed in primary untreated leukemia patient bone marrow samples compared with the expression at the complete remission stage, using reverse transcription-quantitative polymerase chain reaction (RT-qPCR). In addition, a high RSK1 expression (relative expression $\geq 10$ ) was associated with a significantly shorter overall survival $(\mathrm{P}=0.038)$ compared with that in patients with low RSK1 expression (relative expression $<10$ ). The current study also investigated the effect of luteolin, a novel p90 ribosomal S6 kinase (RSK) inhibitor extracted from Reseda odorata L., which shows strong biochemical functions including anti-allergy, anti-inflammation and anti-cancer functions, in MOLM-13 and Kasumi-1 leukemic cells. The cell viability, apoptosis and migration ability analysis were assessed by performing a cell counting kit- 8 assay, Annexin V-FITC/PI double staining and migration filter assay, respectively. The results indicated that luteolin inhibited the growth of the leukemic cell lines through induction of apoptosis, while the migration ability was also suppressed. Overexpression of RSK1 by plasmid transfection was found to decrease the luteolin-induced apoptosis and migration
\end{abstract}

Correspondence to: Dr Zhigang Lu, Department of Hematology, Zhujiang Hospital, Southern Medical University, 253 Industrial Avenue, Haizhu, Guangzhou, Guangdong 501282, P.R. China E-mail: zhiganglu_zhujiang@126.com

Dr Xiuju Wang, Department of Hematology, Sun Yat-Sen Memorial Hospital, Sun Yat-Sen University, 170 Yanjiang Road, Yuexiu, Guangzhou, Guangdong 510120, P.R. China

E-mail: 1219964452@qq.com

*Contributed equally

Key words: luteolin, ribosomal S6 kinase 1, leukemia, apoptosis, migration capabilities. By contrast, knockdown of the RSK1 expression by small interfering RNA appeared to induce the same effect as luteolin on MOLM-13 and Kasumi-1 leukemic cells. In conclusion, these results suggest that luteolin inhibits leukemic cell proliferation and induces apoptosis by inhibition of the RSK1 pathways.

\section{Introduction}

Ribosomal S6 kinases (RSKs) are a family of serine/threonine protein kinases that are directly regulated by extracellular signal-regulated kinase (ERK) signaling. RSK1, a member of the RSK family, was initially identified as an X-linked gene in patients with mental retardation (1-3). Typically, RSK is expressed in the cerebellum during embryogenesis and silenced postnatally. Aberrant RSK signaling is integral for various types of cancer, including breast, colon and renal cancer, as well as melanoma. Li et al (4) observed that the p90 RSK2-cAMP response element-binding protein (CREB) pathway is commonly activated in diverse metastatic human cancer cells. Degen et al (5) also demonstrated this phenomenon, and further observed that overexpression of RSK3 and RSK1 supports cellular proliferation under the PI3K signaling pathway blockade. This occurs through the inhibition of apoptosis and regulation of cellular translation in squamous carcinoma cell through phosphorylation of RSK and eukaryotic translation initiation factor 4B. It is thus reported that RSK1 serves a role in squamous carcinoma cell growth and proliferation. In addition, Cohen et al (6) observed that RSK1 overexpression is associated with sunitinib resistance in renal cell carcinoma cell lines. Elf et al (7) also reported that, although RSK1 is dispensable for BCR-ABL-induced myeloid leukemia, it may be required for the pathogenesis and lineage determination in FLT3-ITD-induced hematopoietic transformation. However, the expression of RSK1 in leukemia remains uncertain.

In the past decades, numerous studies have attempted to identify natural compounds with potential anti-leukemic activity (8-10). Luteolin, also known as 3',4',5',7'-tetrahydroxyflavone, is a flavonoid compound that has a C6-C3-C6 structure and possesses two benzene rings, an oxygen-containing ring and a 2-3 carbon double bond. This compound has been 
found to possess various beneficial properties, including antioxidant (11), anti-inflammatory (12), anti-bacterial (13) and anti-cancer activities (14-17). Recently, Reipas et al (18) reported that luteolin is a novel p90 RSK inhibitor that can be considered as a promising candidate agent for the treatment of liver, lung, breast, colon, prostate, gastric and ovarian cancer, as well as melanoma. In the present study, the effect of Luteolin on AML was investigated.

\section{Materials and methods}

Materials. Luteolin was purchased from Sigma-Aldrich (Oakville, ON, USA) and dissolved in dimethyl sulfoxide to stock concentration of $100 \mathrm{mM}$ at $-20^{\circ} \mathrm{C}$. The Cell Counting kit-8 (CCK-8) and Hoechst 33258 were purchased from Beyotime Institute of Biotechnology (Beijing, China). Lipofectamine RNAiMAX, TRIzol and RT reagents were purchased from Thermo Fisher Scientific, Inc. (Waltham, MA, USA). SYBR Green Supermix was purchased from Takara Bio, Inc. (Otsu, Japan). An Annexin V-FITC/propidium iodide (PI) double staining kit was purchased from Nanjing KeyGen Biotech Co., Ltd. (Nanjing, China). Antibodies were purchased against the phosphorylated forms of RSK1 (Ser221; ab10695; Abcam, Cambridge, MA, USA), B-cell lymphoma (Bcl)-2-associated death promoter (BAD) (Ser112; 9291; Cell Signaling Technology, Inc., Danvers, MA, USA), kidney/brain protein (KIBRA) (Ser947; ab107637; Abcam) and GAPDH (ab8245; Abcam), and were diluted at $1: 1,000$.

Patients and specimens. A total of 30 patients with primary newly-diagnosed acute myeloid leukemia (AML) were enrolled into the present study. The patients underwent consecutive chemotherapy at the Department of Hematology of the Sun Yat-Sen Memorial Hospital (Sun Yat-Sen University, Guangzhou, China) between July 2011 and July 2014. The samples were obtained by bone marrow aspiration prior to initiation of the therapy and after finishing 6 cycles of daunorubicin and cytarabine (DA) therapy, which was defined as the first complete remission (CR) stage (CR1). Each chemotherapy cycle included standard dose cytarabine $\left(100-200 \mathrm{mg} / \mathrm{m}^{2}\right)$ as a continuous infusion for 7 days with daunorubicin hydrochloride $\left(40-60 \mathrm{mg} / \mathrm{m}^{2}\right) \times 3$ once every 28 days. Informed consent was obtained from all patients following a protocol approved by the Ethics Committee of the Sun Yat-Sen Memorial Hospital, Sun Yat-Sen University. AML was evaluated by the World Health Organization classification (19). CR was defined as a bone marrow sample with $<5 \%$ blast cells and a neutrophil count of $>10,000$ cells.

$R T$-qPCR. RSK1 level was detected in MOLM-13, Kasumi-1 and primary AML cells. The primary AML cells were isolated from from $2 \mathrm{ml}$ bone marrow using lymphocyte separation liquid by a density gradient centrifugation method (20). For each clinical sample, mononuclear cells were isolated from $2 \mathrm{ml}$ bone marrow using lymphocyte separation liquid by a density gradient centrifugation method. In order to compare the expression of RSK1 in leukemic cell line, mononuclear cells were isolated from $2 \mathrm{ml}$ peripheral blood of 3 cases using the seam method, and these samples were used as the control group. Total RNA was extracted from the samples using TRIzol reagent, according to the manufacturer's instructions. The cDNA was synthesized using the M-MLV reverse transcriptase kit (Invitrogen; Thermo Fisher Scientific, Inc.). Quantitative analysis of RSK1 mRNA expression was evaluated by qPCR using SYBR ${ }^{\circledR}$ Green One-Step qRT-PCR kit (11736059; Invitrogen; Thermo Fisher Scientific, Inc.), and $\beta$-actin was used as an endogenous control. The sequences of the qPCR primers were as follows: RSK1 forward, 5'-GGT GGTCCTATGGGGTGTTG-3', and reverse, 5'-TCGCCT TCAGAATCAGTGTCA-3'; and $\beta$-actin forward, 5'-TGA AGTGTGACGTGGACATC-3', and reverse, 5'-GGAGGA GCAATGATCTTGAT-3'. All the reactions were performed in a $20 \mu \mathrm{l}$ reaction volume in triplicate. The thermal cycling conditions were as follows: A denaturation step of 31 cycles at $90^{\circ} \mathrm{C}$ for $20 \mathrm{sec}$, an annealing step at $52^{\circ} \mathrm{C}$ for $25 \mathrm{sec}$ and synthesis at $72^{\circ} \mathrm{C}$ for $20 \mathrm{sec}$. The fold changes were calculated through relative quantification with the $2^{-\Delta \Delta C q}$ method.

Cell culture. MOLM-13 and Kasumi-1 cells (Type Culture Collection of the Chinese Academy of Sciences, Shanghai, China) were maintained in RPMI-1640 medium (Gibco; Thermo Fisher Scientific, Inc.) containing 10\% heat-inactivated fetal bovine serum (10437028; Gibco; Thermo Fisher Scientific, Inc.) in a $95 \%$ humidified incubator with $5 \% \mathrm{CO}_{2}$ at $37^{\circ} \mathrm{C}$. The cultures were split every second day by dilution to a concentration of $2 \times 10^{5}$ cells $/ \mathrm{ml}$.

Construction of the RSK1 plasmid and blocking RSK1 expression with small interfering RNA (siRNA). The full-length human RSK1 cDNA sequence was amplified from the cDNA library of MOLM-13 cells by PCR using the following two primers: Sense, 5'-AAG GTA CCA CCA TGG AGC AGG ATC CCA AGC-3', and antisense, 5'-CTC TCG AGT CAC AGG GTG GTG GAT GGC-3'. Taq DNA polymerase (Thermo Fisher Scientific, Inc.) was used for amplification. The RSK1 cDNA sequencing was inserted into the pcDNA3.1 plasmid (Invitrogen; Thermo Fisher Scientific, Inc.). In order to silence RSK1 expression by siRNA, $21 \mathrm{nt}$ complementary RNA with symmetrical 2 nt overhangs was obtained from Shanghai GenePharma Co., Ltd. (Shanghai, China). The DNA sequence was 5'-CCC AAC ATC ATC ACT CTG AAA-3'. The siRNA-RSK1 NC was also transferred into leukemic cells, which had the same number and type of bases as the siRNA-RSK1, but the arrangement was different. The DNA sequence was 5'-CCC AAC CTA ATC TAC CTG AAA-3'. The plasmid and siRNA was transferred into MOLM-13 and Kasumi-1 cells, respectively.

Cell viability assay. MOLM-13 and Kasumi-1 cells were seeded into 96-well culture plates at a density of $1.5 \times 10^{4}$ cells/well in $100 \mu \mathrm{l}$ medium and were treated with different concentrations $(15-60 \mu \mathrm{M})$ of luteolin for 24,48 and $72 \mathrm{~h}$. The pcDNA3.1-RSK1 plasmid was initially constructed, which overexpressed RSK1. Following transfection of cells with pcDNA3.1-RSK1 or empty plasmid for $24 \mathrm{~h}$, the cells were treated with $30 \mu \mathrm{M}$ luteolin, which was the representable value near the half maximal inhibitory concentration $\left(\mathrm{IC}_{50}\right)$, for $24 \mathrm{~h}$. Viable cells were then evaluated with the CCK-8 assay according to the manufacturer's instructions. Briefly, 
CCK-8 solution ( $50 \mu \mathrm{l} /$ well) was added to the cells in 24 -well plates $\left(0.6 \times 10^{4}\right.$ cells/well), and the samples were incubated at $37^{\circ} \mathrm{C}$ for $4 \mathrm{~h}$. Subsequently, the optical density of each well was read at $450 \mathrm{~nm}$ using a microplate reader (ELx800 absorbance reader; BioTek Instruments, Inc., Winooski, VT, USA). The viability was assessed with the following equation: Viability $(\%)=$ Experimental $/$ Control $\times 100 \%$.

Cell apoptosis assay. Apoptotic and dead cell counts were performed using a FITC-labeled Annexin V and PI staining by flow cytometry. The cells were collected and resuspended in binding buffer at a concentration of $3 \times 10^{6} / \mathrm{ml}$. Next, $100 \mu \mathrm{l}$ cell suspension was added to $5 \mu 1$ Annexin V-FITC and $10 \mu \mathrm{l}$ PI, and the sample was mixed for $15 \mathrm{~min}$ in the dark at room temperature. Subsequently, $400 \mu 1$ phosphate-buffered saline was added to the solution. A FACScan instrument (BD Biosciences, Franklin Lakes, NJ, USA) was used to count the cells $\left(1 \times 10^{3}\right)$ at an excitation wavelength of $490 \mathrm{~nm}$ and determine the cell apoptosis. CellQuest software version 5.1 (BD Biosciences) was used for data collection and processing.

Western blot analysis. SDS Lysis Buffer (P0013G; Beyotime Institute of Biotechnology) was added to MOLM-13 and Kasumi-1 cells to isolate the proteins. Protein concentration was detected using a bicinchoninic acid kit (P0009; Beyotime Institute of Biotechnology). For each western blot sample, $20 \mu \mathrm{g}$ protein was load onto each lane and resolved through $12 \%$ SDS-PAGE, and then electrophoretically transferred to a polyvinylidene difluoride membrane (EMD Millipore, Billerica, MA, USA). Next, the membrane was probed with primary antibodies against human RSK1 (Ser221; ab10695, Abcam), BAD (Ser112; 9291; Cell Signaling Technology, Inc.) and KIBRA (Ser947; ab107637; Abcam), with GAPDH (ab8245; Abcam) used as the internal control. The antibodies were diluted 1:1,000 and incubated at room temperature for $2 \mathrm{~h}$. Next, the membrane was incubated with horseradish peroxidase-conjugated goat anti-rabbit secondary antibody (ab6721; Abcam; dilution, 1:5,000) at room temperature for $1.5 \mathrm{~h}$. The membranes were visualized with SignalFire ${ }^{\mathrm{TM}}$ ECL Reagent (\#6883; Cell Signaling Technology, Inc.), and the band intensity was measured by Quantity One software 3.0 (Bio-Rad Laboratories, Inc. Hercules, CA, USA).

Migration assay. The migration of MOLM-13 and Kasumi-1 cells was assayed using a Boyden chamber (Qilinbeier Bio Co., Haimen, China). Cells were treated with different concentrations of luteolin and then plated at $1 \times 10^{6}$ cells $/ \mathrm{ml}$ in the upper chamber, with 10\% RPMI-1640 medium added to the lower chamber. After incubating for $24 \mathrm{~h}$, non-migration cells were removed from the top well, while the bottom cells were collected and counted by trypan blue exclusion assay in triplicate.

Statistical analysis. All computations were carried out using the SPSS v18.0 for Windows (SPSS, Inc., Chicago, IL, USA). Data were expressed as mean \pm standard deviation. One-way analysis of variance was used to analyze the differences, and Kaplan-Meier survival analysis was used to analyze patient survival rate. $\mathrm{P}<0.05$ was considered to indicate a statistically significant difference.
Table I. Association of RSK1 expression with clinicopathological characteristics of 30 acute myeloid leukemia patients.

\begin{tabular}{|c|c|c|c|c|}
\hline \multirow[b]{2}{*}{ Clinical variables } & \multirow[b]{2}{*}{ Number } & \multicolumn{2}{|c|}{$\begin{array}{c}\text { RSK1 } \\
\text { expression (n) }\end{array}$} & \multirow[b]{2}{*}{ P-value } \\
\hline & & $\begin{array}{l}\text { Low } \\
(<10)\end{array}$ & $\begin{array}{l}\text { High } \\
(\geq 10)\end{array}$ & \\
\hline Gender & & & & 0.559 \\
\hline Male & 12 & 8 & 4 & \\
\hline Female & 18 & 10 & 8 & \\
\hline Age (years) & & & & 0.884 \\
\hline$<60$ & 17 & 10 & 7 & \\
\hline$\geq 60$ & 13 & 8 & 5 & \\
\hline Leukocytes (cells/ $\mu 1)$ & & & & 0.146 \\
\hline$>10,000$ & 15 & 7 & 8 & \\
\hline$\leq 10,000$ & 15 & 11 & 4 & \\
\hline Hemoglobin (g/dl) & & & & 0.667 \\
\hline$>80$ & 16 & 9 & 7 & \\
\hline$\leq 80$ & 14 & 9 & 5 & \\
\hline Platelet (cells/ $\mu \mathrm{l}$ ) & & & & 0.447 \\
\hline$>25,000$ & 20 & 11 & 9 & \\
\hline$\leq 25,000$ & 10 & 8 & 2 & \\
\hline FAB & & & & 0.227 \\
\hline M1-6 & 29 & 18 & 11 & \\
\hline M7 & 1 & 0 & 1 & \\
\hline MRD cells $(\%)$ & & & & 0.002 \\
\hline$<0.25$ & 14 & 12 & 2 & \\
\hline$\geq 0.25$ & 16 & 6 & 10 & \\
\hline
\end{tabular}

RSK1, ribosomal S6 kinase 1; FAB, French-American-Britain; MRD, minimal residual disease.

\section{Results}

High expression of RSK1 is observed in newly diagnosed $A M L$ cases. Aberrant RSK signaling is integral for several types of cancer, such as breast cancer and melanoma. In the present study, bone marrow samples from $30 \mathrm{AML}$ patients (12 males and 18 females) at the newly-diagnosed and CR stages were analysed for RSK1 expression using the RT-qPCR method. The clinicopathological characteristics of the 30 AML patients are shown in Table I. The expression of RSK1 was found to be associated with the French-American-Britain (FAB) subtype and the percentage of minimal residual disease (MRD) cells. There were no statistically significant differences in other clinical features, including the patient gender and age, as well as the leukocyte, hemoglobin and platelet counts, between individuals with high and low RSK1 expression. Fig. 1A shows a markedly higher expression of RSK1 in newly diagnosed patients compared with that in patients at the CR1 stage. Furthermore, the prognostic significance of RSK1 expression was assessed in the 30 adult AML patients according to the clinical follow-up records. Kaplan-Meier survival analysis indicated that the high RSK1 

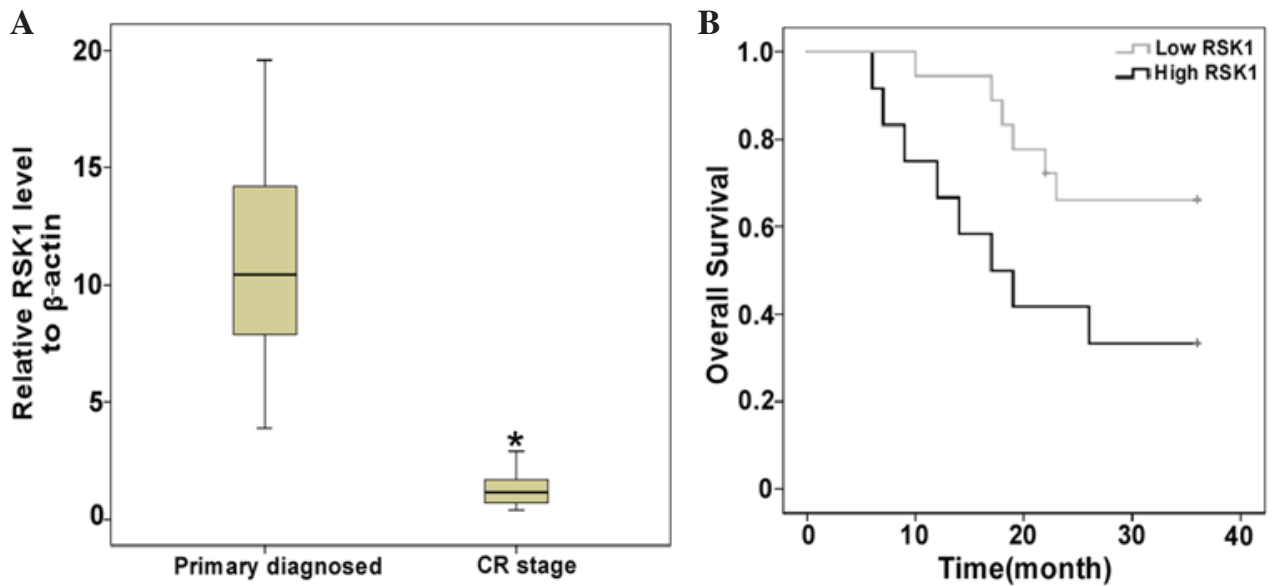

Figure 1. RSK1 is overexpressed in primary AML patients. (A) Reverse transcription-quantitative polymerase chain reaction analysis of the RSK1 mRNA transcript levels in bone marrow mononuclear cell samples of 30 adult AML patients prior to treatment (newly diagnosed) and at the CR stage after 6 cycles of standard chemotherapy ( $\mathrm{P}<0.005)$. (B) Kaplan-Meier survival analysis of $30 \mathrm{AML}$ patients, comparing patients with high and low RSK1 expression (P=0.038). AML, acute myeloid leukemia; RSK1, ribosomal S6 kinase 1.

expression group tended to have a shorter overall survival (OS) compared with that of the low RSK1 expression group $(\mathrm{P}=0.038$; Fig. 1B).

Effect of luteolin on viability, chemosensitivity and apoptosis in leukemia cells. RSKs serve a role in various cellular processes, including gene expression, cell survival, apoptosis and proliferation. The present study verified that, compared with normal mononuclear cells, RSK1 overexpression was observed in the leukemic cell lines MOLM-13 and Kasumi-1 (Fig. 2A). In order to investigate whether luteolin effects leukemic cells through RSK1, the cell viability of MOLM-13 and Kasumi-1 were first investigated upon treatment with luteolin. The luteolin concentration range used in the present experiment was $15-60 \mathrm{nM}$ for 24,48 and $72 \mathrm{~h}$. The $\mathrm{IC}_{50}$ for each concentration was determined and averaged for the triplicate experiments, and the final $\mathrm{IC}_{50}$ values of luteolin on MOLM-13 and Kasumi-1 cells were found to be 34.75 and $34.05 \mu \mathrm{M}$, respectively. Luteolin treatment resulted in a marked reduction of cell proliferation in a dose-dependent manner (Fig. 2B).

To understand the mechanism by which luteolin caused viability loss in MOLM-13 and Kasumi-1 cells, apoptosis experiments were performed (Fig. 2C). The resultant viability loss was accompanied by decreased phosphorylation of proteins targeted by RSKs, such as BAD $(21,22)$, which is a proapoptotic protein (Fig. 2D). Collectively, the current findings indicate that luteolin was able to induce the viability loss, while apoptosis of MOLM-13 and Kasumi-1 cells was associated with RSK1-associated apoptotic injury.

Effect of luteolin on migration of leukemia cells. Leukocytes migrate into and out of blood vessels at multiple points during their development and maturation, and during immune surveillance. Migration is also an important part of leukemia progression. To determine the influence of luteolin on in vitro cell migration, the Boyden chamber assay was performed in MOLM-13 and Kasumi-1 cells. As shown in Fig. 3A, the results indicated that there was significantly less migration through the basement membrane following treatment with luteolin, in a dose-dependent manner. Cell migration of MOLM-13 and Kasumi-1 cells drove us to examine the possible biological functions of luteolin in leukemic cells, particularly the effect of this treatment on cell migration (Fig. 3B). The results demonstrated that the phosphorylated level of KIBRA also decreased in a dose-dependent manner.

Overexpression of $R S K 1$ reverses the effects of luteolin treatment on leukemic cells. To examine the role of RSK1 in the generation of luteolin-induced functional responses, MOLM-13 and Kasumi-1 leukemia cells were treated in the presence of luteolin with the RSK1-overexpressing plasmid or control plasmid. The overexpression of RSK1 mRNA and protein expression levels were evaluated by RT-qPCR and western blot analysis, respectively (Fig. 4A and B). As determined by RT-qPCR, the RSK1 mRNA level was upregulated by 15 -fold, and the protein level in the RSK1 overexpression group was 3-fold greater than that of the control. The cell viability was also determined by CCK- 8 assays, as shown in Fig. 4C. For the luteolin with RSK1-overexpressing plasmid group, transfection with the RSK1-overexpressing plasmid significantly counteracted the effect of luteolin on cell viability when compared with the luteolin alone group (Fig. 4C). Similarly, when induction of apoptosis was assessed, the apoptotic ratio of the luteolin and RSK1-overexpressing group was found to be significantly decreased (Fig. 4D), with a similar effect observed in the migration ability of cells (Fig. 4E).

Knocking down of RSK1 has the same effects on leukemic cells as luteolin treatment. To further verify whether RSK1 serves an important role in the luteolin-induced functional responses, the expression of RSK1 was blocked by siRNA transfection in MOLM-13 and Kasumi-1 leukemia cells. The siRNA silenced the expression of RSK1 on the mRNA and protein levels, as evaluated by RT-qPCR and western blot analysis, respectively (Fig. 5A and B). Following transfection with the siRNA-RSK1 in the leukemic cell lines, the cell viability was determined by CCK- 8 assay. The results demonstrated that the viability of the siRNA-RSK1 group 
A

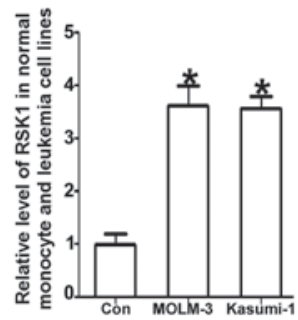

B

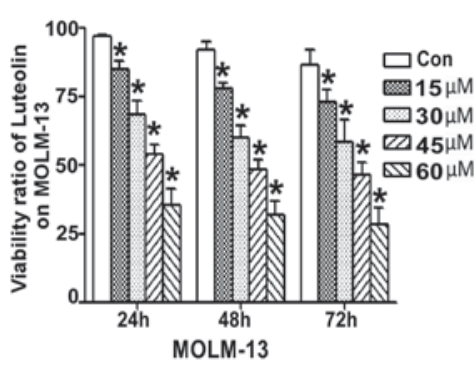

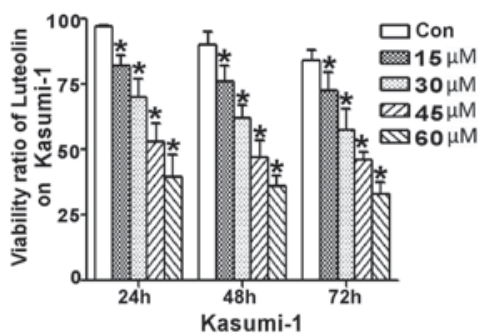

C
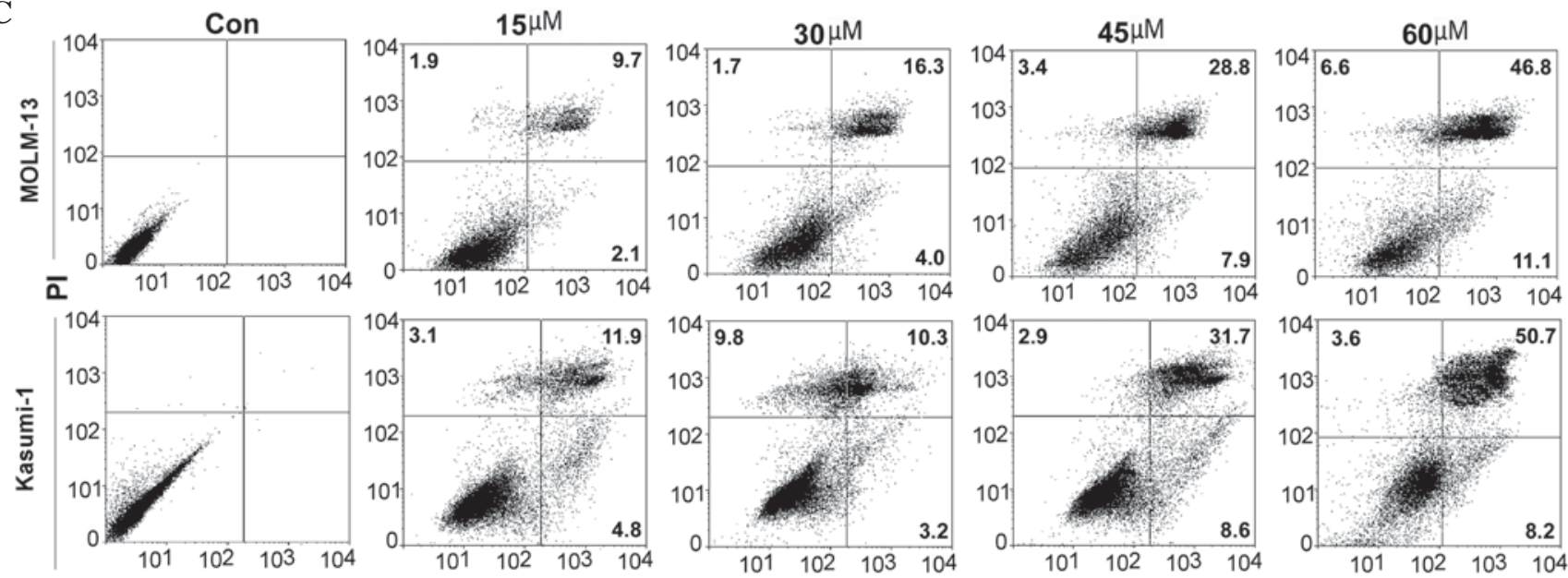

AnnexinV

D

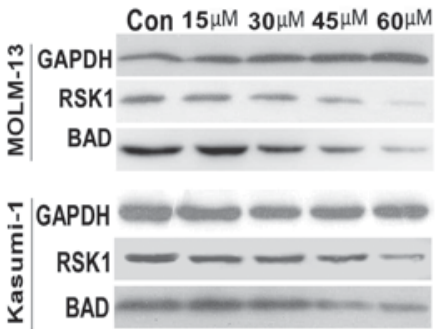

Figure 2. Luteolin treatment regulates the cell proliferation and apoptosis in MOLM-13 and Kasumi-1 cells. (A) RSK1 was significant higher in leukemic cell lines compared with the normal controls. (B) Following exposure to various concentrations (15-60 $\mu \mathrm{M}$ ) of luteolin for 24, 48 and $72 \mathrm{~h}$, the viability of MOLM-13 and Kasumi- 1 cells was determined using the cell counting kit- 8 assay. The viability ratio decreased in a dose-dependent manner ("P<0.01 vs. untreated control). (C) Apoptosis of MOLM-13 and Kasumi-1 cells treated with 15-60 $\mu \mathrm{M}$ luteolin for $24 \mathrm{~h}$ was investigated by flow cytometry. Higher concentrations of luteolin treatment resulted in increased apoptosis in a dose-dependent manner. (D) Protein levels of RSK1 and phosphorylated BAD (Ser ${ }^{112}$ ) were decreased in a dose-dependent manner in leukemic cells treated with luteolin, as observed by western blotting. RSK1, ribosomal S6 kinase 1; BAD, Bcl-2-associated death promoter; Bcl, B-cell lymphoma; PI, propidium iodide; Con, control.

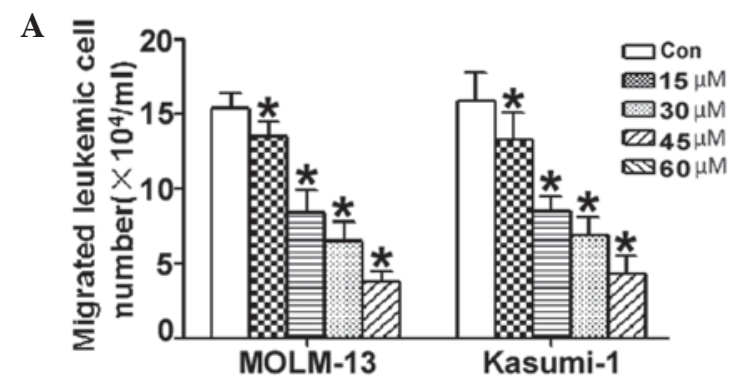

B

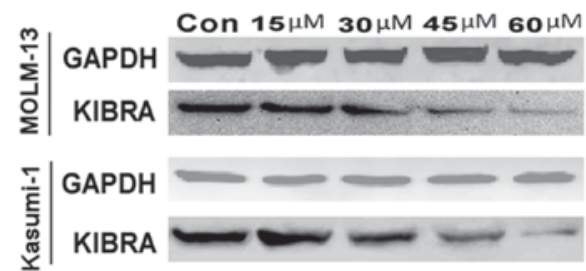

Figure 3. Luteolin treatment reduces cell migration. MOLM-13 and Kasumi-1 cells treated with $15-60 \mu \mathrm{M}$ luteolin for $24 \mathrm{~h}$ were investigated by migration assay with a Boyden chamber. (A) Lower chamber cells were collected and counted by trypan blue exclusion assay, showing reduced migration upon luteolin treatment, in a dose-dependent manner. (B) Upper chamber cells were collected and analyzed for the level of phosphorylated KIBRA (Ser ${ }^{947}$ ), which was found to decrease in a dose-dependent manner. "P<0.05 vs. untreated control. RSK1, ribosomal S6 kinase 1; KIBRA, kidney/brain protein; Con, control.

was significantly lower compared with that of the untreated control and NC groups (Fig. 5C). Similarly, when induction of apoptosis was assessed, the results identified that the apoptotic ratio of the siRNA group was significantly increased (Fig. 5D). By contrast, the migration ability decreased markedly upon silencing of RSK1 with siRNA (Fig. 5E). 
A

C
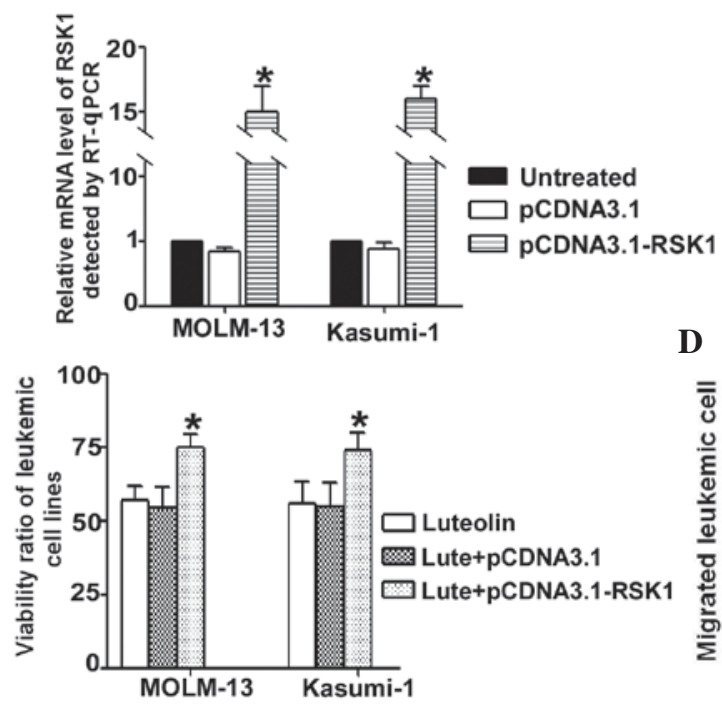

$\mathbf{E}$
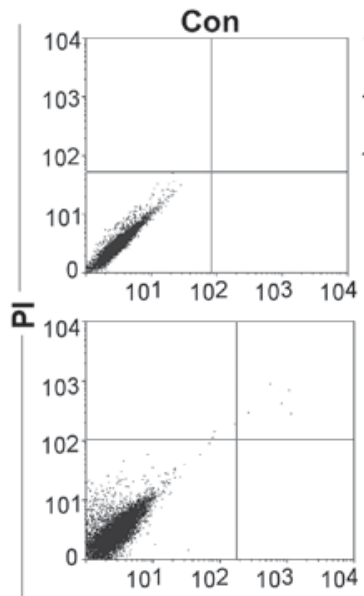
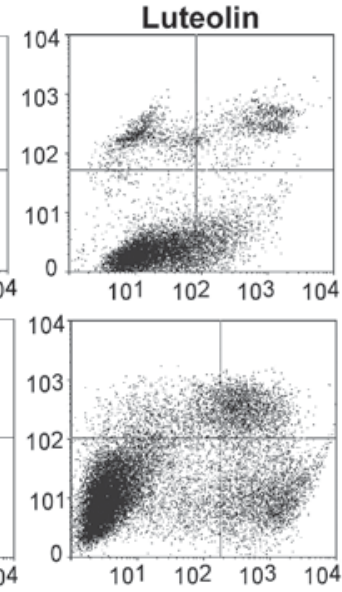

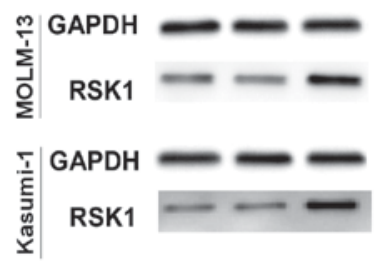

D

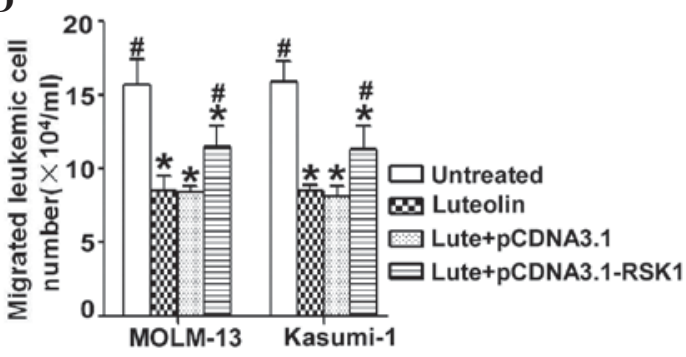

Lute+pCDNA3.1
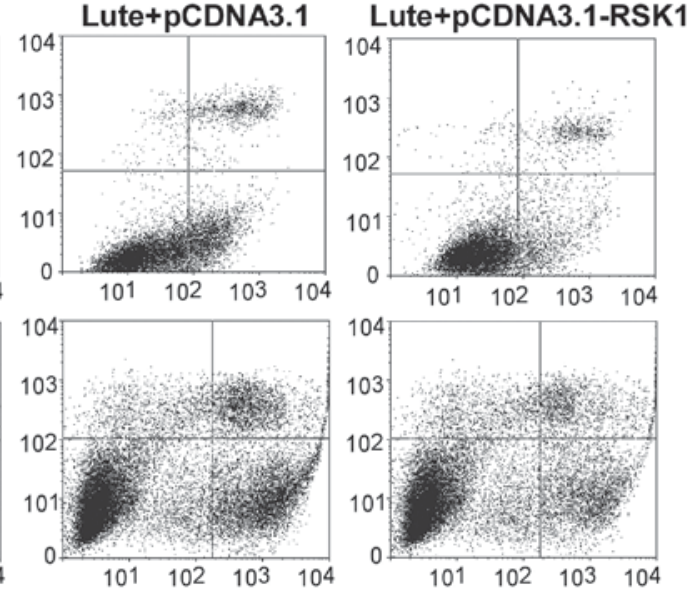

AnnexinV

Figure 4. Transfection with an RSK1-overexpressing plasmid for $24 \mathrm{~h}$ reverses the luteolin-induced apoptosis in MOLM-13 and Kausmi-1 cells. Cells were treated with luteolin alone $(30 \mu \mathrm{M})$, or combined with a RSK1-overexpressing plasmid or empty vector for $24 \mathrm{~h}$. (A) mRNA and (B) protein expression levels of RSK1 were evaluated by RT-qPCR and western blot analysis, respectively. (C) Cell viability detected by the cell counting kit-8 method indicated that RSK1 overexpression reversed the effects of luteolin on the cells, with a significantly higher viability ratio detected (" $\mathrm{P}<0.01$ vs. luteolin alone). (D) Cell migration assay indicated that transfection with the RSK1-overexpressing plasmid reversed the effects of luteolin (" $\mathrm{P}<0.01$ vs. untreated group; ${ }^{*} \mathrm{P}<0.01$ vs. luteolin alone group). (E) Changes in the apoptotic ratio of MOLM-13 and Kasumi-1, as detected by flow cytometry. In the RSK1-overexpressing group, the apoptotic ratio was markedly reduced compared with the other two groups. RSK1, ribosomal S6 kinase 1; Lute, luteolin; Con, control; PI, propidium iodide; RT-qPCR, reverse transcription-quantitative polymerase chain reaction.

\section{Discussion}

Proliferation, survival and migration of cells are regulated by different intracellular signaling pathways. Among these, the Ras/mitogen-activated protein kinase kinase (MEK)/ERK signaling pathway has been established (23). Novel treatments targeting the RSK, PI3K/Akt and MEK/ERK signaling pathways are currently under evaluation in numerous types of tumors in clinical trials (24-26).

AML is an aggressive hematological disorder characterized by an abnormal increase of immature hematopoietic progenitor cells known as 'blasts', which lose their ability to differentiate normally and continue to proliferate. The downstream effectors MEK and Erk1/2 are constitutively activated in the majority of AML cases. The present study focused on the members of the RSK family of Ser/Thr kinases, which are downstream effectors of the MAPK/ERK pathway, whose activities regulate cellular effectors that promote cell growth, survival and migration (27).
This kinase family consists of four human isoforms (RSK1-4), which possess a $73-80 \%$ amino acid identity and have the same general structure, and two structurally associated homologues (1). RSKs function downstream of MEK, ERK and protein kinase $\mathrm{C}$, and are frequently activated in various types of cancer, including leukemia, and this activation may be triggered by steroids, insulin, epidermal growth factor and estrogen. Previous studies have demonstrated that RSK1 is overexpressed in prostate (28), breast (29) and colon cancer tissues (30).

In the present study, the association between the expression of RSK1 and the outcome of 30 cases AML was analyzed. Bone marrow mononuclear cells from 30 patients with AML were isolated, and the mRNA level of RSK1 was detected by RT-qPCR. The percentage of MRD cells is the main independent prognostic factor for leukemia, and the expression of RSK1 was found to be closely associated with the MRD percentage in the present study. Regarding the FAB subtypes of patients in the current study, only one patient had an M7 

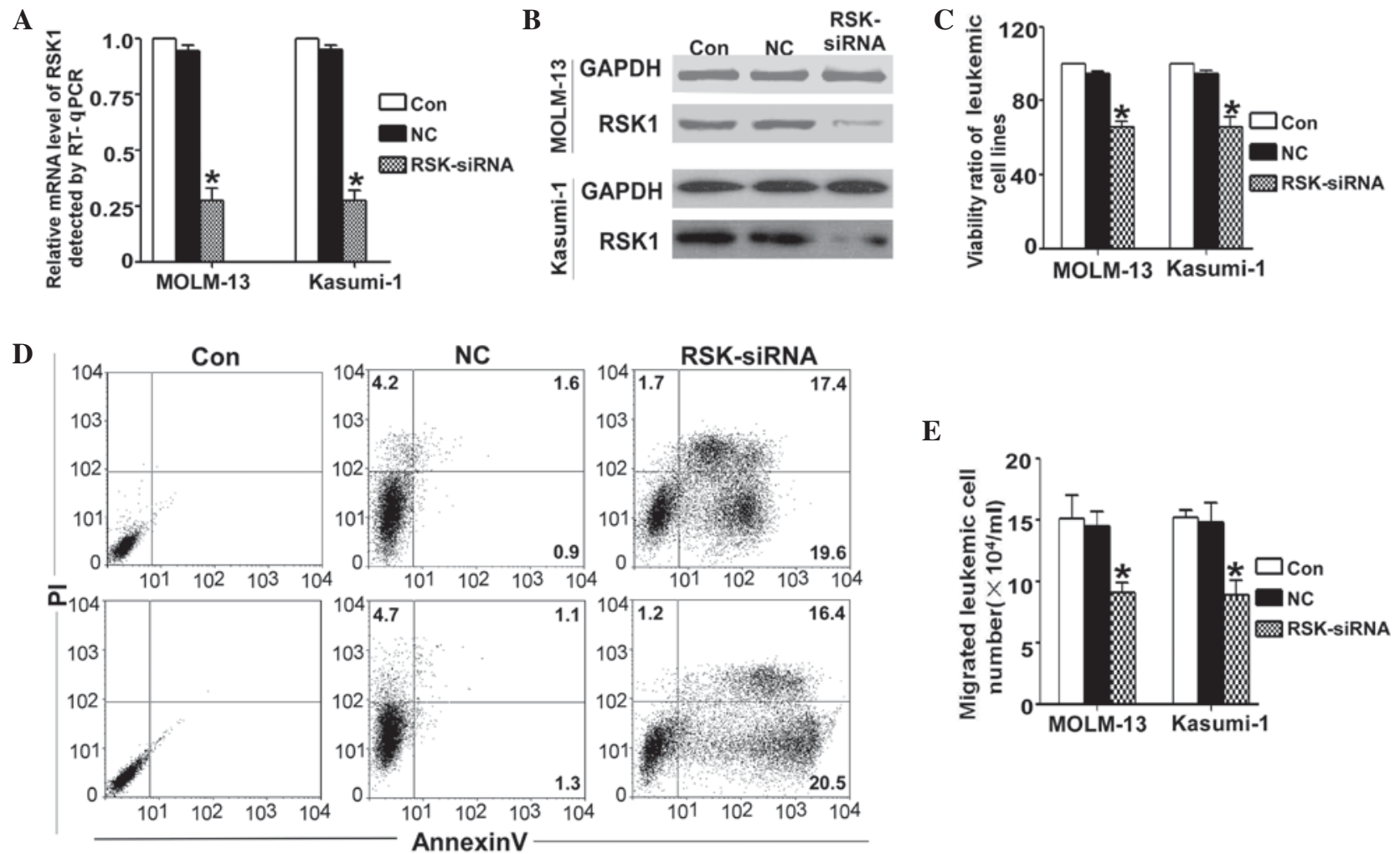

Figure 5. Silencing of RSK1 by transfection with siRNA-RSK1 for $24 \mathrm{~h}$ had a similar effect to that of luteolin treatment on MOLM-13 and Kausmi-1 cells. (A) mRNA and (B) protein expression levels of RSK1 were RT-qPCR and western blot analysis, respectively. (C) Cell viability was detected by the cell counting kit-8 method following transfection with siRNA-RSK or NC for $24 \mathrm{~h}$. The viability ratio was significant lower compared with that of the two control groups. (D) Changes in the apoptotic ratio of MOLM-13 and Kasumi-1 cells, as detected by flow cytometry. In the siRNA-RSK1 group, the apoptotic ratio was markedly higher compared with that of the other two groups. (E) Cell migration ability of the two cell lines, showing that the siRNA-RSK1 group presented markedly lower migration compared with the control group. "P $\mathrm{P} 0.001$ vs. the untreated control group. RSK1, ribosomal S6 kinase 1; Con, untreated control; NC, negative control; PI, propidium iodide; siRNA, small interfering RNA; RT-qPCR, reverse transcription-quantitative polymerase chain reaction.

subtype. Future studies should thus include a larger number of cases and samples. According to the present results, the expression of RSK1 was not associated with the clinical characteristics of the AML patients, such as hyperleukocytosis, severe anemia, thrombocytopenia and older age ( $>60$ years). High expression of RSK1 was also observed in the newly diagnosed AML samples compared with the CR stage (Fig. 1A). Kaplan-Meier survival analysis described that a higher RSK1 level was correlated with a shorter OS of patients (Fig. 1B).

AML is an extremely heterogeneous disease with a variable long term survival rate ranging between 20 and $90 \%$. The major interventions of conventional medicine failed to have its effects because of its side effects. An increasing number of studies have been focusing on Chinese herb extracts, with certain compounds recently reported as molecular targeted anti-cancer drugs, such as luteolin, a novel inhibitor of RSK. Luteolin has been the focus of clinical-translational interest in anticancer treatment (31-33). In the present study, the effects of pharmacological inhibition of the RSK1 inhibitor, luteolin, on leukemic cell lines were investigated. As shown in Fig. 2A, luteolin exhibited potent suppressive effects on MOLM-13 and Kasumi- 1 cells in a dose-dependent manner, with $\mathrm{IC}_{50}$ values of 34.75 and $34.05 \mu \mathrm{M}$, respectively.

Cancer cells acquire alternations for enhanced survival and become apoptosis-resistant to anticancer therapies. Therefore, the induction of apoptosis can be an effective approach for inhibiting uncontrolled cell proliferation and survival of malignant tumor cells. Compared with the untreated control cells, luteolin treatment led to cell apoptosis in the present study (Fig. 1B). A mechanism through which RSK interacts to cause apoptosis is the alteration of BAD phosphorylation, thus triggering its proapoptotic functions. BAD is a unique $\mathrm{BH} 3$-only protein, since its functions are tightly regulated by serine phosphorylation (34). In the hypophosphorylated form, BAD interacts with Bcl-2 or Bcl-extra large to neutralize their anti-apoptotic functions. RSK has been shown to phosphorylate BAD on the Ser112 site. The present study observed that luteolin was able to induce BAD dephosphorylation in a dose-dependent manner. Therefore, it is likely that luteolin inhibited RSK1 with the hypophosphorylated form of BAD.

It has been reported that RSKs serve important roles in cell migration as well (35). Yang et al (36) reported that RSK1 specifically phosphorylates KIBRA, a memory performance-associated protein, and also modulates its migratory activity in MDA-MB-231 breast cancer cells. Recently, KIBRA was demonstrated to be a positive regulator of the Hippo signaling pathway activity in mammalian cells (37). The present study demonstrated that luteolin, an RSK1 inhibitor, impaired the migration of MOLM-13 and Kasumi-1 cells in a dose-dependent manner through dephosphorylation of KIBRA. Thus, there results indicate that KIBRA, the downstream factor of RSK1, serves an important role in the mobility of leukemia cells. The current study next explored whether RSK1 expression is a key factor in the effect of luteolin 
treatment on leukemic cell proliferation and migration. An RSK1-overexpressing plasmid was transfected in leukemic cell lines for $24 \mathrm{~h}$, and cells were then treated with luteolin. The viability, apoptosis and migration analysis results supported that RSK1 serves an important role in luteolin-modulated cell proliferation and migration of AML cells.

In conclusion, the current pooled analysis demonstrated that RSK1 mRNA expression was significantly higher in primary AML samples compared with the expression at the CR stage following standard chemotherapy. In addition, a shorter survival time was correlated with high RSK1 expression. Luteolin treatment triggered RSK-dependent antileukemic responses with dephosphorylation of BAD or KIBRA. Therefore, luteolin, a novel RSK inhibitor, has significant anti-leukemia effects in vitro and may function as a possible therapeutic in the future; however, further investigation in clinical trials is first required to confirm these results.

\section{Acknowledgements}

The present study was supported by grants from the Natural Science Foundation of Guangdong Province (grant nos. S2012010008748 and S2013010014715) and the National Natural Science Foundation of China (grant no. 81400103).

\section{References}

1. Lara R, Seck1 MJ and Pardo OE: The p90 RSK family members: Common functions and isoform specificity. Cancer Res 17: 5301-5308, 2013

2. Romeo Y,Zhang X and Roux PP: Regulation and function of the RSK family of protein kinases. Biochem J 441: 553-569, 2012.

3. Kostenko S, Shiryaev A, Dumitriu G, Gerits N and Moens U: Cross-talk between protein kinase A and the MAPK-activated protein kinases RSK1 and MK5. J Recept Signal Transduct Res 31: 1-9, 2011.

4. Li D, Jin L, Alesi GN, Kim YM, Fan J, Seo JH, Wang D, Tucker M, Gu TL, Lee BH, et al: The prometastatic ribosoma S6 kinase 2-cAMP response element-binding protein (RSK2-CREB) signaling pathway up-regulates the actin-binding protein fascin-1 to promote tumor metastasis. J Biol Chem 288: 32528-32538, 2013.

5. Degen M, Barron P, Natarajan E, Widlund HR and Rheinwald JG: RSK activation of translation factor eIF4B drives abnormal increases of laminin $\gamma 2$ and MYC protein during neoplastic progression to squamous cell carcinoma. PLoS One 8: e78979, 2013.

6. Cohen JD, Babiarz JE, Abrams RM, Guo L, Kameoka S, Chiao E, Taunton J and Kolaja KL: Use of human stem cell derived cardiomyocytes to examine sunitinib mediated cardiotoxicity and electrophysiological alterations. Toxicol Appl Pharmacol 257: 74-83, 2011.

7. Elf S, Blevins D, Jin L, Chung TW, Williams IR, Lee BH, Lin JX, Leonard WJ, Taunton J, Khoury HJ and Kang S: p90RSK2 is essential for FLT3-ITD-but dispensable for BCR-ABL-induced myeloid leukemia. Blood 117: 6885-6894, 2011.

8. Sak K and Everaus H: Chemomodulating effects of flavonoids in human leukemia cells. Anticancer Agents Med Chem 15: $1112-1126,2015$.

9. Morceau F, Chateauvieux S, Orsini M, Trecul A, Dicato M and Diederich M: Natural compound and pharmaceuticals reprogram leukemia cell differentiation pathways. Biotechnol Adv 33 (6 Pt 1): 785-797, 2015.

10. Humisto A, Herfindal L, Jokela J, Karkman A, Bjornstad R, Choudhury RR and Sivonen K: Cyanobacteria as a source for novel anti-leukemic compound. Curr Pharm Biotechnol 17: 78-91, 2016.

11. Xu Y, Zhang J, Liu J, Li S, Li C, Wang W, Ma R and Liu Y: Luteolin attenuate the d-galactose-induced renal damage by attenuation of oxidative stress and inflammation. Nat Prod Res 29: 1078-1082, 2015.
12. Sung $J$ and Lee $J$ : Anti-inflammatory activity of butein and luteolin through suppression of $\mathrm{NFKB}$ activation and induction of heme oxygenase-1. J Med Food 18: 557-564, 2015.

13. Eumkeb G, Siriwong S and Thumanu K: Synergistic activity of luteolin and amoxicillin combination against amoxicillin-resistant Escherichia coli and mode of action. J Photochem Photobiol B 117: 247-253, 2012.

14. Kim YS, Kim SH, Shin J, Harikishore A, Lim JK, Jung Y, Lyu HN, Baek NI, Choi KY, Yoon HS and Kim KT: Luteolin suppresses cancer cell proliferation by targeting vaccinia-related kinase 1. PLoS One 9: e109655, 2014.

15. Park SH, Ham S, Kwon TH, Kim MS, Lee DH, Kang JW, Oh SR and Yoon DY: Luteolin induces cell cycle arrest and apoptosis through extrinsic and intrinsic signaling pathways in MCF-7 breast cancer cells. J Environ Pathol Toxicol Oncol 33: 219-231, 2014.

16. Chian S, Li YY, Wang XJ and Tang XW: Luteolin sensitizes two oxaliplatin-resistant colorectal cancer cell lines to chemotherapeutic drugs via inhibition of the Nrf2 pathway. Asian Pac J Cancer Prev 15: 2911-2916, 2014.

17. Chen KC, Chen CY, Lin CR, Yang TY, Chen TH, Wu LC and Wu CC: Luteolin attenuates TGF- $\beta 1$-induced epithelial-mesenchymal transition of lung cancer cells by interfering in the PI3K/Akt-NF-кB-Snail pathway. Life Sci 93: 924-933, 2013.

18. Reipas KM, Law JH, Couto N, Islam S, Li Y, Li H, Cherkasov A, Jung $\mathrm{K}$, Cheema AS, Jones SJ, et al: Luteolin is a novel p90 ribosomal S6 kinase (RSK) inhibitor that suppresses Notch4 signaling by blocking the activation of Y-box binding protein-1 (YB-1). Oncotarget 4: 329-345, 2013.

19. Falini B, Tiacci E, Martelli MP, Ascani S and Pileri SA: New classification of acute myeloid leukemia and precursor-related neoplasms: Changes and unsolved issues. Discov Med 10: 281-292, 2010.

20. Flygare J, Kiefer T, Miyake K, Utsugisawa T, Hamaguchi I, Da Costa L, Richter J, Davey EJ, Matsson H, Dahl N, et al: Deficency of ribosomal protein S19 in CD34+ cells generated by siRNA blocks erythroid development and mimics defected seen in Diamond-Blackfan anemia. Blood 105: 4627-4634, 2005.

21. Sun D, Huang J, Zhang Z, Gao H, Li J, Shen M, Cao F and Wang H: Luteolin limits infarct size and improves cardiac function after myocardium ischemia/reperfusion injury in diabetic rats. PLoS One 7: e33491, 2012.

22. Lee WR, Shen SC, Lin HY, Hou WC, Yang LL and Chen YC: Wogonin and fisetin induce apoptosis in human promyeloleukemic cells, accompanied by a decrease of reactive oxygen species, and activation of caspase 3 and $\mathrm{Ca}^{2+}$-dependent endonuclease. Biochem Pharmacol 63: 225-236, 2002.

23. Friday BB and Adjei AA: Advances in targeting the Ras/Raf/MEK/Erk mitogen-activated protein kinase cascade with MEK inhibitors for cancer therapy. Clin Cancer Res 14: 342-346, 2008.

24. Crudden C, Ilic M, Suleymanova N, Worrall C, Girnita A and Girnita L: The dichotomy of the Insulin-like growth factor 1 receptor: RTK and GPCR: Friend or foe for cancer treatment? Growth Horm IGF Res 25: 2-12, 2015.

25. Sabine VS, Crozier C, Brookes CL, Drake C, Piper T, van de Velde CJ, Hasenburg A, Kieback DG, Markopoulos C, Dirix L, et al: Mutational analysis of PI3K/AKT signaling pathway in tamoxifen exemestane adjuvant multinational pathology study. J Clin Oncol 32: 2951-2958, 2014.

26. Johnson AS, Crandall H, Dahlman K and Kelley MC: Preliminary results from a prospective trial of preoperative combined BRAF and MEK-targeted therapy in advanced BRAF mutation-positive melanoma. J Am Coll Surg 220: 581-593.e1, 2015.

27. Dent P: Arsenic in leukemia: A RSKy business. Cancer Biol Ther 14: 871-872, 2013.

28. Yu G, Lee YC, Cheng CJ, Wu CF, Song JH, Gallick GE, Yu-Lee LY, Kuang J and Lin SH: RSK promotes prostate cancer progression in bone through ING3, CKAP2, and PTK6-mediated cell survival. Mol Cancer Res 13: 348-357, 2015.

29. Stratford AL, Reipas K, Hu K, Fotovati A, Brough R, Frankum J, Takhar M, Watson P, Ashworth A, Lord CJ, et al: Targeting p90 ribosomal S6 kinase eliminates tumor-initiating cells by inactivating Y-box binding protein-1 in triple-negative breast cancers. Stem Cells 30: 1338-1348, 2012.

30. Goulet AC, Watts G, Lord JL and Nelson MA: Profiling of selenomethionine responsive genes in colon cancer by microarray analysis. Cancer Biol Ther 6: 494-503, 2007. 
31. Sato Y, Sasaki N, Saito M, Endo N, Kugawa F and Ueno A Luteolin attenuates doxorubicin-induced cytotoxicity to mcf-7 human breast cancer cells. Biol Pharm Bull 38: 703-709, 2015

32. Bumke-Vogt C, Osterhoff MA, Borchert A, Guzman-Perez V, Sarem Z, Birkenfeld AL, Bähr V and Pfeiffer AF: The flavones apigenin and luteolin induce FOXO1 translocation but inhibit gluconeogenic and lipogenic gene expression in human cells. PLoS One 9: e104321,2014.

33. Chian S, Thapa R, Chi Z, Wang XJ and Tang X: Luteolin inhibits the Nrf2 signaling pathway and tumor growth in vivo. Biochem Biophys Res Commun 447: 602-608, 2014.

34. Thimmaiah KN, Easton JB and Houghton PJ: Protection from rapamycin-induced apoptosis by insulin-like growth factor-I is partially dependent on protein kinase $\mathrm{C}$ signaling. Cancer Res 70: 2000-2009, 2010.
35. Czaplinska D, Turczyk L, Grudowska A, Mieszkowska M, Lipinska AD, Skladanowski AC, Zaczek AJ, Romanska HM and Sadej R: Phosphorylation of RSK2 at Tyr529 by FGFR2-p38 enhances human mammary epithelial cells migration. Biochim Biophys Acta 1843: 2461-2470, 2014.

36. Yang S, Ji M, Zhang L, Chen Y, Wennmann DO, Kremerskothen J and Dong J: Phosphorylation of KIBRA by the extracellular signal-regulated kinase (ERK)-ribosomal S6 kinase (RSK) cascade modulates cell proliferation and migration. Cell Signal 26: 343-351, 2014.

37. Xiao L, Chen Y, Ji M and Dong J: KIBRA regulates Hippo signaling activity via interactions with large tumor suppressor kinases. J Biol Chem 286: 7788-7796, 2011. 